\section{SPECIALISE IN SEDATION}

The Eastman Dental Institute's Postgraduate Certificate in Dental Sedation and Pain Management is designed to equip clinicians with the knowledge, skills, practical training and confidence to provide effective and safe sedation in line with current best practice.

Commencing in May 2011 with six days of lectures and problem-based learning, practical training follows with practitioners administering conscious sedation to patients under

\section{WEARABLE BLEACHING DEVICE}

The iPower is the first wearable and portable thermal diffusion bleaching device in the world and is safe, powerful, cost effective and easy to use.

The iPower comes in a handy box the size of an A4 folder. The patient wears the complete system and can walk around while having the treatment. The iPower has a Thermal Diffuser Head that hooks onto a retractor in the patient's mouth. So it moves with the patient's head and maintains the correct position at all times.

The iPower is the first bleaching device without any visible light irradiating onto the teeth and the oral cavity. Instead, the wy10 iPower uses Thermal Diffusion Technology to deliver optimum results in the safest possible way. It regulates and maintains the thermal energy in the bleaching gel on the surface of the tooth. This accurately controlled environment makes for a safe and pain-free treatment.

Tests show that the iPower halves treatment time. The thermal head contains a tiny heating panel with a supervision. Didactic training continues in November 2011 to provide graduates with immersion training in medical emergencies and simulated sedation complications as well as an introduction to paediatric sedation and other topics.

A final day will be held in spring 2012 and to review all patients treated and cover latest updates.

Applications for the courses are encouraged by 11 March 2011 and are offered on a first come first served basis.

\section{Reader response number 53}

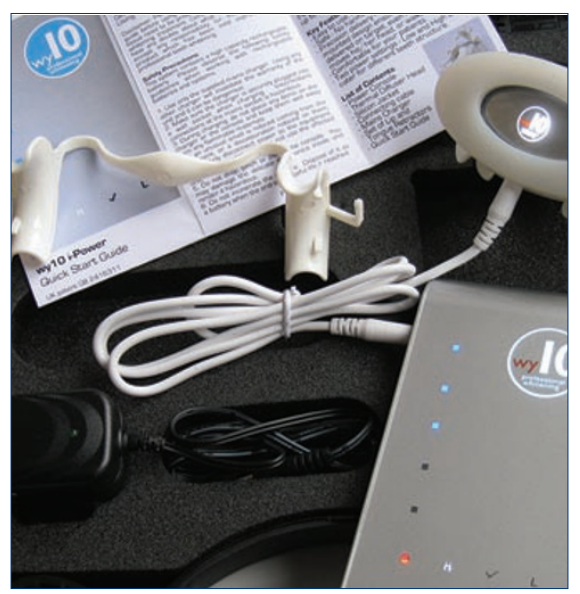

sensor and delivers an accurate amount of thermal energy onto the bleaching gel painted on the patient's teeth. A thermal gradient thus created in the gel increases the diffusion rate of bleaching agent into the enamel structure, speeding up the entire process.

A ten-minute timer ensures that the device is foolproof and used with wy10 gel, teeth are left up to ten shades whiter with no sensitivity. The device also has energy saving features so is environmentally friendly.

Reader response number 54

\section{CUTTING EDGE AIR CONTROL}

Hitachi's S-Series room air conditioning range, the RAS SX8, can help dental practices strengthen their fight against cross infection.

The S-Series destroys $99.9 \%$ of airborne viruses and bacteria, while delivering the most comfortable level of heating and cooling. As well as creating a healthier environment, the sleek, wall-mounted S-Series is also highly energy efficient and cost effective to run.

Each unit generates ionised mist from the air in the room and also automatically cleans its micro-mesh stainless filter once the air conditioning operation has ended, removing dust from the filter and collecting it in a dust box. Its internal cleaning operation controls mould growth as well.

The S-Series delivers cutting edge temperature control - monitoring and controlling all aspects of the air humidity and air quality to enable the complete air conditioning service.

The DC Inverter technology allows a room to reach the required temperature rapidly ensuring maximum user comfort. The inverter technology then adjusts the speed of the system's 'EcoScroll' compressor to an energy saving mode, in order to maintain the desired temperature as efficiently as possible. Reader response number 55

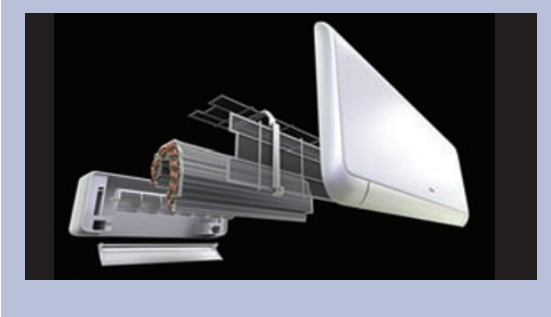

\section{QUALITY WITHOUT COMPROMISE}

Castellini's range of dental units captures its design philosophy, blending high level performance with flexibility and ergonomics.

With a unit to suit every budget, the new range includes the Puma Eli 5 which has a wealth of stand- ard features, offering efficiency and operative convenience. The Skema 5 combines sturdiness with practicality with advanced hygiene systems, design features facilitating daily cleansing operations and high performance instruments.

The Skema 6 offers flexible and functional design, creating an ideal working space allowing the dentist to work unhindered. With extensive travel arms and everything within reach, dentists can focus on the patient while reducing physical stress.

The DUO Plus combines innovative solutions, unrivalled hygiene systems and perfectly integrated instruments, embodying total quality without any compromise.

Reader response number 56 